\title{
WHOLE CHROMOSOME ANALYSIS OF HEIGHT IN WHEAT
}

\author{
J. W. SNAPE, C. N. LAW and A. J. WORLAND \\ Plant Breeding Institute, Trumpington, Cambridge CB2 2LQ
}

Received 25.vi.76

\begin{abstract}
SUMMARY
The genetical architecture of height in the cross between the wheat varieties Chinese Spring and Cappelle-Desprez is examined using crosses involving the complete set of single chromosome substitution lines of Cappelle-Desprez into Chinese Spring. Seventeen of the 21 chromosome pairs exhibit genetical variation with respect to this character and in addition to additive and dominance effects, epistatic effects of an additive $\times$ additive type are shown to be an important component of the variation.

The details of the genetical architecture revealed by whole chromosome analysis on the one hand and conventional generation means analysis on the other are very similar. However, the whole chromosome analysis reveals a much greater complexity and in particular shows that the heterosis exhibited in the $F_{1}$ between the parents is explained by the dispersion of increasing chromosomes between the parents with directional dominance for increasing loci, combined with between chromosome interactions.

Estimation of the effects of individual chromosomes is attempted but a satisfactory model is not found. Nevertheless, tentative predictions as to the consequences of new chromosomal combinations are made on the basis of the best fitting model.
\end{abstract}

\section{Introduction}

The ability of bread wheat, Triticum aestivum $(2 n=6 x=42)$ to tolerate aneuploidy has led to the development of cytological techniques whereby single pairs of chromosomes can be transferred from one variety to another. Such chromosome substitution lines provide a powerful tool for the genetical analysis of the wheat crop, particularly with respect to genetical variation for loci controlling quantitative characters of agronomic interest.

Grossing procedures using such lines have been described by Law (1966, 1968, 1972), Aksel (1967) and Snape, Law and Worland (1975), which enable the detailed genetical analysis of chromosomal differences with respect to quantitative characters to be carried out. These methods, named the Triparental crosses 1 and 2, allow the individual additive, dominance and interaction effects of single chromosomes to be detected and, in the absence of non-homologous chromosome interactions (epistasis) the effects of chromosome substitutions can be unambiguously estimated and the value of new chromosomal cornbinations predicted.

Law and Worland ( $1973 a$ and $b$ ) have extended these analyses to completely specify the genetical differences between two varieties, Chinese Spring and Hope, by carrying out the complete set of Triparental 2 crosses using the complete set of Hope into Chinese Spring substitutions. From their analysis of the character's height and 250 grain weight they identified the chromosomal locations of loci showing variation with respect to these characters and using estimates of these effects they were able to predict 
minimal limits to selection and the frequencies of genotypes with desirable combinations of these characters.

The present paper is concerned with extending these analyses further to examine the genetical differences with respect to height between the varieties Chinese Spring and Cappelle-Desprez using the complete sct of Cappelle-Desprez substitutions into Chinese Spring. Final plant height is a character to which considerable attention has been paid by plant breeders and the cross between these varieties is particularly intercsting in this respect sincc previous experiments have shown that although thesc varieties are of comparable height, they exhibit significant positive heterosis in the $F_{1}$ and $F_{2}$ generations. Thus the detailed genetical analysis using chromosome substitution lines can help to elucidate the genetical basis of this heterosis. Further the analysis of individual chromosome effects can be compared with the conventional analysis of generation means of the parental, $\mathrm{F}_{1}, \mathrm{~F}_{2}$ and backcross generations (Mather and Jinks, 1971) where balanced summed effects of all chromosomes are considered, and the relative efficiencies of these analyses in elucidating the genetical architecture of this character examined.

\section{Materials AND methods}

The complete set of 21 single chromosome substitution lines of CappelleDesprcz into Chinese Spring, developed by the authors at the Plant Breeding Institute, were crossed to the two parental lines and the $F_{1}$ between them to produce the complete set of extended Triparental 2 crosses (Snape, Law and Worland, 1975). Random crosses between the substitution lines were also carricd out to produce a total of 39 Triparental cross 1 generations. The parental varieties and the $\mathrm{F}_{1}$ were selfed and intercrossed to produce the parcntal, $F_{1}, F_{2}$ and backcross generations.

The rcsults of these crossing procedures produced 129 different generations as shown in table 1 . These generations were sown in plots in each of

TABLE 1

\section{Generations grown}

Generation No. plots/line/block grown

Cappelle-Desprcz
Chinese Spring $(\mathrm{CS})$
Cappelle-Desprez $\times \mathrm{CS} \mathrm{F}_{1}$
Cappclle-Desprez $\times \mathrm{CS} \mathrm{F}_{2}$
$\mathrm{~F}_{1} \times$ Cappelle-Desprez
$\mathrm{F}_{1} \times$ Chinese Spring
21 Substitution lines
21 Substitution lines $\times$ Cappelle-Desprez
21 Substitution lines $\times \mathrm{CS}$
21 Substitution lines $\times \mathrm{F}_{1}$
39 Substitution lines $\times$ Substitution lines

5
5
15
34
14
11
4
$3-5$
$3-5$
$6-10$
$2-5$

two randomised blocks in the field. The experiment was sown in early February so that the vernalisation requirements of both varicties would be satisfied. Each plot consisted of a row of 11 plants with a $10 \mathrm{~cm}$ spacing between plants and a $30 \mathrm{~cm}$ spacing between rows. The number of plots per line per block were variable, depending on the amount of seed available 
from each cross and are shown in table l. Measurements of final plant height were made on each individual plant at harvest.

\section{Detegtion of genetical EFFeGts}

Block differences and block interactions were found to constitute only a small proportion of the variation between plot means so that in the analyses presented the data have been averaged over plots and blocks for each generation.

The generation means for the extended Triparental 2 crosses are shown in table 2.

TABLE 2

Triparental 2 crosses: generation means: heights $(\mathrm{cm})$

$\begin{array}{ccccc}\text { Chromosome } & \begin{array}{c}\text { Substitution } \\ \text { lines }\end{array} & \begin{array}{c}\text { Substitution } \\ \text { lines } \times \text { CS }\end{array} & \begin{array}{c}\text { Substitution lines } \\ \times \text { Cappelle Desprez }\end{array} & \begin{array}{c}\text { Substitution } \\ \text { lines } \times F_{1}\end{array} \\ \text { 1A } & 106 \cdot 7 & 110 \cdot 7 & 117 \cdot 1 & 115 \cdot 9 \\ \text { 1B } & 109 \cdot 9 & 106 \cdot 1 & 118 \cdot 7 & 114 \cdot 4 \\ \text { 1D } & 110 \cdot 9 & 105 \cdot 9 & 119 \cdot 1 & 117 \cdot 8 \\ \text { 2A } & 106 \cdot 8 & 109 \cdot 4 & 117 \cdot 0 & 115 \cdot 1 \\ \text { 2B } & 110 \cdot 0 & 11 \cdot \cdot 3 & 118 \cdot 9 & 115 \cdot 9 \\ \text { 2D } & 110 \cdot 3 & 114 \cdot 1 & 119 \cdot 4 & 117 \cdot 7 \\ \text { 3A } & 104 \cdot 4 & 110 \cdot 4 & 115 \cdot 7 & 111 \cdot 5 \\ \text { 3B } & 103 \cdot 7 & 106 \cdot 5 & 113 \cdot 7 & 113 \cdot 6 \\ \text { 3D } & 106 \cdot 5 & 110 \cdot 7 & 119 \cdot 0 & 116 \cdot 7 \\ \text { 4A } & 106 \cdot 1 & 108 \cdot 8 & 113 \cdot 4 & 113 \cdot 7 \\ \text { 4B } & 107 \cdot 9 & 110 \cdot 8 & 115 \cdot 7 & 112 \cdot 8 \\ \text { 4D } & 117 \cdot 1 & 113 \cdot 4 & 128 \cdot 3 & 121 \cdot 6 \\ \text { 5A } & 103 \cdot 4 & 109 \cdot 4 & 118 \cdot 1 & 114 \cdot 3 \\ \text { 5B } & 112 \cdot 3 & 108 \cdot 4 & 119 \cdot 9 & 116 \cdot 4 \\ \text { 5D } & 118 \cdot 6 & 112 \cdot 5 & 124 \cdot 1 & 122 \cdot 9 \\ \text { 6A } & 112 \cdot 4 & 111 \cdot 2 & 121 \cdot 7 & 116 \cdot 3 \\ \text { 6B } & 98 \cdot 4 & 111 \cdot 6 & 120 \cdot 7 & 117 \cdot 7 \\ \text { 6D } & 108 \cdot 5 & 112 \cdot 2 & 119 \cdot 7 & 118 \cdot 3 \\ \text { 7A } & 106 \cdot 0 & 113 \cdot 2 & 114 \cdot 8 & 116 \cdot 3 \\ \text { 7B } & 108 \cdot 5 & 112 \cdot 3 & 119 \cdot 6 & 118 \cdot 1 \\ \text { 7D } & 109 \cdot 6 & 111 \cdot 9 & 119 \cdot 7 & 116 \cdot 5\end{array}$

Chinese Spring $=107 \cdot 3$. Cappelle-Desprez $=103 \cdot 4$. Chinese Spring $\times$ Cappelle $F_{1}=119 \cdot 3$

\section{(i) Additive and within chromosome interaction effects}

Comparisons between the means of the generations derived from the complete set of Triparental 2 crosses allow the identification of the chromosomes showing genetical variation with respect to height (Law, 1972). The estimates of the additive and within chromosome interaction (dominance) effects obtained for each substitution line are shown in table 3.

Five chromosomes exhibit significant additive variation, namely $4 \mathrm{D}, 5 \mathrm{~B}$, $5 \mathrm{D}, 6 \mathrm{~A}$ and $6 \mathrm{~B}$. The sign of the estimate indicates whether the presence of the substituted chromosome increases $(+)$ or decreases $(-)$ the height of the recipient, Chinese Spring. As can be seen, of the five significant estimates, four are positive and one negative. However, considering the estimates of all 21 chromosomes there is considerable dispersion of the increasing chromosomes between the parents, with 13 chromosome 
substitutions increasing the height of the recipient and eight decreasing its height.

Turning to the estimates of the within-chromosome interactions, seven chromosomes exhibit significant effects, namcly 1A, 2D, 3A, 3D, 5A, 6B, 7A. All these estimates are positive, so that potence is predominantly in one direction, namely in favour of chromosomes which are acting to increase height relative to their homologues. Surprisingly however, only one of these chromosomes, that is $6 \mathrm{~B}$, was detected as having a significant additive

TABLE 3

Triparental 2 crosses: estimates of additive, dominance and interaction effects

\begin{tabular}{|c|c|c|c|}
\hline Chromosome & $\begin{array}{l}\text { Additive effect } \\
\qquad(\mathrm{cm})\end{array}$ & $\begin{array}{l}\text { Within chromosome } \\
\text { interaction }(\mathrm{cm})\end{array}$ & $\begin{array}{l}\text { Between chromosome } \\
\text { interaction }(\mathrm{cm})\end{array}$ \\
\hline $1 \mathrm{~A}$ & $-0 \cdot 29$ & $3.72 *$ & $1 \cdot 81$ \\
\hline $1 \mathrm{~B}$ & $1 \cdot 35$ & $-2 \cdot 51$ & $-4 \cdot 46^{*}$ \\
\hline $1 \mathrm{D}$ & $1 \cdot 84$ & $-3 \cdot 24$ & $-5 \cdot 32$ \\
\hline $2 \mathrm{~A}$ & -0.25 & $2 \cdot 40$ & $0 \cdot 30$ \\
\hline $2 B$ & $1 \cdot 39$ & $2 \cdot 66$ & $0 \cdot 84$ \\
\hline $2 \mathrm{D}$ & $1 \cdot 51$ & $5 \cdot 29 * * *$ & 3.89 \\
\hline $3 \mathrm{~A}$ & $-1 \cdot 46$ & $4 \cdot 56^{*}$ & $2 \cdot 43$ \\
\hline $3 \mathrm{~B}$ & $-1 \cdot 79$ & $1 \cdot 05$ & $-2 \cdot 75$ \\
\hline $3 \mathrm{D}$ & -0.37 & $3.81 *$ & 3.88 \\
\hline $4 \mathrm{~A}$ & $0 \cdot 61$ & $2 \cdot 17$ & $-3 \cdot 12$ \\
\hline $4 B$ & $0 \cdot 30$ & $3 \cdot 24$ & -0.62 \\
\hline $4 \mathrm{D}$ & $4 \cdot 93 * * *$ & $1 \cdot 21$ & $5 \cdot 29$ \\
\hline $5 \mathrm{~A}$ & $-1 \cdot 94$ & $4 \cdot 11 * *$ & $4 \cdot 79$ \\
\hline $5 B$ & $2 \cdot 49 * * *$ & $-1 \cdot 32$ & $-3 \cdot 26$ \\
\hline $5 \mathrm{D}$ & $5 \cdot 65 * * *$ & -0.38 & $-1 \cdot 25$ \\
\hline $6 \mathrm{~A}$ & $2 \cdot 56 * * *$ & $1 \cdot 35$ & $1 \cdot 19$ \\
\hline $6 \mathrm{~B}$ & $-4 \cdot 42 * * *$ & $8.79 * * *$ & $14 \cdot 55 * * *$ \\
\hline $6 \mathrm{D}$ & $0 \cdot 63$ & $4 \cdot 28$ & $4 \cdot 00$ \\
\hline $7 \mathrm{~A}$ & $-0 \cdot 62$ & $6 \cdot 59 * * *$ & $2 \cdot 72$ \\
\hline $7 \mathrm{~B}$ & $0 \cdot 60$ & $4 \cdot 46$ & $4 \cdot 16$ \\
\hline $7 \mathrm{D}$ & $1 \cdot 18$ & $3 \cdot 43$ & $2 \cdot 63$ \\
\hline
\end{tabular}

effect. Thus, chromosome substitution lincs which are not, in fact, significantly different from the recipient in height, are nevertheless exhibiting genetical variation with respect to this character. Three explanations are possible for this significant overdominance. First, "true" overdominance may be exhibited by the loci on these chromosomes, that is $h>d$, in the terminology of Mather and Jinks (1971). Secondly, non-homologous chromosone interactions may be present which arc acting to decrease the additive effects, and thirdly, the presence of more than one locus on each chromosome which are linked in the repulsion phase. These two latter explanations are not mutually exclusive but in the present case they cannot be differentiated between or separated from the overdominance hypothesis without further analysis.

\section{(ii) Between non-homologous chromosome interaction effects}

From the results of the crossing procedures described above, two separate tests of bctween non-homologous chromosome interactions (or epistatic 
effects) are possible. The first, using the generations derived from the Triparental 2 crosses detects the summed effects of interactions of each of the chromosomes with all other chromosomes of the complement. These estimates are given in table 3 , and have also been given in an earlier paper (Snape, Law and Worland, 1975). Only two chromosomes, namely 1B and $6 \mathrm{~B}$ are detected as showing significant interactions. Of these, the former is negative and the latter positive. The overdominance exhibited by $6 \mathrm{~B}$ may thus, in part at least, be due to epistatic effects.

The extension of the Triparental 2 crossing procedure to include crosses with the $F_{1}$ between the parents allows an examination of the types of interaction present in this set of crosses. These results were presented in

TABLe 4

Triparental 1 crosses: estimates of between chromosome interaction effects

\begin{tabular}{|c|c|c|c|}
\hline Cross & istimate $(\mathrm{cm})$ & Cross & Estimate $(\mathrm{cm})$ \\
\hline \multicolumn{2}{|c|}{ Between chromosomes within } & $2 \mathrm{~A} \times 6 \mathrm{~A}$ & -0.42 \\
\hline \multicolumn{2}{|c|}{ homoeologous groups } & $2 \mathrm{~B} \times 4 \mathrm{~A}$ & $-5 \cdot 47$ \\
\hline $2 \mathrm{~A} \times 2 \mathrm{~B}$ & -3.89 & $2 \mathrm{~B} \times 6 \mathrm{~A}$ & $-2 \cdot 98$ \\
\hline $3 \mathrm{~B} \times 3 \mathrm{D}$ & $-1 \cdot 75$ & $2 B \times 6 B$ & $-9 \cdot 02 * *$ \\
\hline $3 \mathrm{~B} \times 3 \mathrm{~A}$ & $-3 \cdot 32$ & $2 \mathrm{D} \times 3 \mathrm{~A}$ & $-11 \cdot 30 * * *$ \\
\hline $4 \mathrm{~A} \times 4 \mathrm{D}$ & $-7 \cdot 76 *$ & $2 \mathrm{D} \times 5 \mathrm{~A}$ & $-12 \cdot 55 * * *$ \\
\hline \multirow{2}{*}{\multicolumn{2}{|c|}{$\begin{array}{l}\text { (ii) Between chromo } \\
\text { different homoeo }\end{array}$}} & $3 \mathrm{~B} \times 5 \mathrm{~B}$ & $2 \cdot 13$ \\
\hline & & $3 \mathrm{~B} \times 6 \mathrm{D}$ & $-4 \cdot 08$ \\
\hline $1 \mathrm{~A} \times 7 \mathrm{D}$ & $-6 \cdot 59 *$ & $3 \mathrm{D} \times 7 \mathrm{~B}$ & $-7 \cdot 00 *$ \\
\hline $1 \mathrm{~B} \times 5 \mathrm{~A}$ & $-1 \cdot 90$ & $3 \mathrm{D} \times 7 \mathrm{D}$ & $-4 \cdot 70$ \\
\hline $1 \mathrm{~B} \times 4 \mathrm{D}$ & $-0 \cdot 68$ & $4 \mathrm{~B} \times 6 \mathrm{D}$ & $-6 \cdot 10$ \\
\hline $1 \mathrm{~B} \times 6 \mathrm{~B}$ & $2 \cdot 18$ & $4 B \times 7 A$ & $-10 \cdot 71$ \\
\hline $1 \mathrm{~B} \times 6 \mathrm{D}$ & $1 \cdot 10$ & $4 \mathrm{~B} \times 7 \mathrm{D}$ & $-5 \cdot 54$ \\
\hline $1 \mathrm{D} \times 2 \mathrm{D}$ & -0.66 & $4 \mathrm{D} \times 7 \mathrm{~B}$ & $-8.93^{*}$ \\
\hline $1 \mathrm{D} \times 3 \mathrm{D}$ & 0.82 & $5 \mathrm{~A} \times 7 \mathrm{D}$ & $-13 \cdot 63 * * *$ \\
\hline $1 \mathrm{D} \times 6 \mathrm{~B}$ & $-2 \cdot 91$ & $5 B \times 7 A$ & $-4 \cdot 50$ \\
\hline $1 \mathrm{D} \times 6 \mathrm{~B}$ & $2 \cdot 77$ & $5 B \times 7 B$ & $-4 \cdot 75$ \\
\hline $1 \mathrm{D} \times 7 \mathrm{~B}$ & 1.85 & $6 \mathrm{~A} \times 7 \mathrm{~A}$ & $-7 \cdot 02 *$ \\
\hline $2 \mathrm{~A} \times 3 \mathrm{~A}$ & $-6 \cdot 80^{*}$ & $6 \mathrm{~B} \times 7 \mathrm{~A}$ & -2.63 \\
\hline $2 \mathrm{~A} \times 3 \mathrm{~A}$ & $-5 \cdot 73^{*}$ & $6 \mathrm{D} \times 7 \mathrm{~A}$ & $-8 \cdot 23 * * *$ \\
\hline $2 \mathrm{~A} \times 5 \mathrm{~B}$ & $-5 \cdot 45$ & $6 \mathrm{D} \times 7 \mathrm{D}$ & $-5 \cdot 50$ \\
\hline $2 \mathrm{~A} \times 5 \mathrm{D}$ & $-9 \cdot 47 *$ & & \\
\hline
\end{tabular}

the earlier paper and indicated that chromosomes other than $1 \mathrm{~B}$ and $6 \mathrm{~B}$ were involved in interactions and that these interactions were almost exclusively of an additive $\times$ additive (i) type. Furthermore, although the " net" interactions of individual chromosomes were ambi-directional, there was a predominance of negative interactions so that the epistasis is primarily of a duplicate type (Mather, 1967).

Individual interactions between pairs of non-homologous chromosomes can be detected using the generation means of the Triparental 1 crosses. The estimates derived from the 39 random cross carried out are shown in table 4.

Turning first to interactions between chromosomes within the same homoeologous group, only the cross $4 \mathrm{~A} \times 4 \mathrm{D}$ exhibits significant interaction. However, all estimates are negative, which taken together with the positive estimates of the within chromosome interactions (table 3 ), indicates again 
a duplicate type of gene interaction (Law, 1972). The substitution lines of Hope into Chinese Spring also showed this type of genetical architecture (Law and Worland, 1973a and $b$ ) which they related to the hexaploid nature of the wheat genome and the functional identity of chromosomes within homoeologous groups. However, looking at the cstimates of interactions between chromosomes of different homoeologous groups, again all significant estimates are negative and oppose the additive and within chromosome estimates. This indicates that duplicate interactions are an important component of the genetical architecture of height in this cross, over and above chromosome homoeology. Furthermore, chromosomes of all homoeologous groups and genomes are involved in interactions although the largest interactions are between chromosomes of the $\mathrm{A}$ and $\mathrm{D}$ genomes.

It is intcresting to compare the Triparcntal 1 and 2 tests for cpistasis. From the results here it would appear that the Triparental 2 tests arc particularly affected by the balancing effects of ambi-directional interactions and chromosomes which are showing large individual interactions are only detected as such through crosses between the respective substitution lines.

These results also show that the overdominance exhibited by the substitution lines showing significant within chromosome interactions must, at least in part, be due to epistatic effects, since in all cases these chromosomes exhibit significant between chromosome interactions. Furthermore, the chromosomes showing the largest overdominance are also the chromosomes showing the largest interactions, although $6 \mathrm{~B}$ is an exception to this.

The above results demonstrate the complexity of the genetical control of height in the cross between these two wheat varieties. Seventeen chromosomes of the complement are detected as contributing to the variation between the substitution lines, the exceptions being chromosomes 1D, 3B, $4 \mathrm{~A}$ and $4 \mathrm{~B}$. The large epistatic component of the variation indicates that the estimatcs of the individual additive and within chromosome interaction effects will contain epistatic components and will be, on their own, of little predictive value. The unambiguous estimation of these effects requires, thereforc, a more complex gcnetical analysis, where the individual additive, dominance and interaction parameters can be allowed for simultaneously.

\section{Estimation of Genetical effects}

The parameters described by Law (1972) can be used to define the genetical effects of each chromosome. Here $d$ is defined as the additive difference between homologous chromosomes, $h$ as the dominance and/or non-allelic interaction within a chromosome, and $i, j$ and $l$ as the additive $\times$ additive, additive $\times$ within chromosome interaction, and within-chromosome interaction $\times$ within-chromosome interaction parameters, respectively. These parameters are defined from a common origin $m$ which in the present case represents the mean of all possible inbred lines derived by random rcassortment of the 21 chromosome pairs. This is equivalent to the Foometric (Van der Veen, 1959) but excludes recombination within chromosomes.

Models including these parameters, describing the effects of all 21 pairs of homologous chromosomes, can be fitted to the observed generation means by the method of weighted least squares. Models of increasing complexity are specified sequentially until an adequate model is obtained, and this then provides estimates of the genetical parameters describing the 
variation in this set of substitution lines. These can then be used to examine the genetical architecture of the character and to predict the consequences of new chromosomal combinations.

In the present experiment a total of 129 generation means are available for the fitting process. Thus, up to 128 parameters can be specified whilst still allowing degrees of freedom to test the goodness of fit of the model. If epistatic effects were absent then only 43 parameters would be needed to completely specify the variation, that is, $m, 21 d$ 's and $21 h$ 's. But as was seen from the above tests for epistasis and also can be seen from the failure to fit this model to the observed generation means (table 5), epistasis is an important component of the genetical architecture of this character.

To completely specify all the genetical effects relating to the 21 substitution lines when epistasis is present, and ignoring tri-chromosomal and higherorder interactions requires 883 parameters, that is, $m, 21 d$ 's, $21 h$ 's, $210 i$ 's, $420 j$ 's and $210 l$ 's. Obviously to completely specify an adequate model in the present case requires great simplification of these parameters. One method of simplifying the number of interaction parameters is to exploit

TABLE 5

Model fitting on generation means: goodness of fit

\begin{tabular}{lrcc}
\multicolumn{1}{c}{ Model } & \multicolumn{1}{c}{$\chi_{\mathbf{2}}$} & d.f. & $\mathbf{P}$ \\
1. Minimal model, 43 parameters & $252 \cdot 0$ & 86 & $<0.001$ \\
2. Complete model, 99 parameters & $806 \cdot 0$ & 30 & $<0.001$ \\
3. Best fitting model, 74 parameters & 86.6 & 54 & $0 \cdot 01<0.001$
\end{tabular}

the known genetical architecture of wheat and to fit interactions which relate to variation within and between homoeologous groups only. Law and Worland (1973a and $b$ ) used such a model on the data from the Hope into Chinese Spring substitution set. Interactions were restricted to within homoeologous groups and were set such that $i_{\mathrm{AB}}=i_{\mathrm{AD}}=i_{\mathrm{BD}}, l_{\mathrm{AB}}=$ $l_{\mathrm{AD}}=l_{\mathrm{BD}}, j_{\mathrm{AB}}=j_{\mathrm{AD}}=j_{\mathrm{BD}}=j_{\mathrm{BA}}=j_{\mathrm{DA}}=j_{\mathrm{DB}}$. They imposed the further restraint that $i=l$. This model required only 14 interaction parameters (two per homoeologous group) and the full 57 parameter model was found to give an adequate description of the variation in height in those substitution lines. Since in the present experiment between homoeologous group interactions are also present, these can be likewise completely specified in terms of only 42 further parameters.

The fit of this full model on the present data is shown in table 5. The $\chi^{2}$ for goodness of fit is highly significant showing that this 99 parameter model is incapable of explaining the observed variation. Other models completely specifying the interaction parameters in simplified terms were also tried and none was able to account for the variation. Thus, either the assumption of equality of interactions is wrong or higher order interactions are present. The data in table 3 would suggest that the former is the case. It would appear, therefore, that it is necessary to specify individual interactions if an adequate model is to be found. It is possible to simplify the requirements of the model somewhat since the extended Triparental 2 analysis shows the interactions to be primarily of the $i$ type. However, even here, there are 210 possible $i$ type parameters which can be specified and there are a large number of permutations of these that can take up the 
degrees of frecdom available and the limitation of 85 parameters may not be sufficient to obtain a fit.

Nevertheless, a number of models containing different combinations were fitted to the observed generation means and the addition of individual interaction parameters greatly improved the fit over the 43 parameter model. Although no model was found which adequately explained the observed variation a model containing $29 i$ 's and $3 l$ 's was found to give the smallest $\chi^{2}$ for goodness of fit (table 5). This model accounts for 83 per cent of the genotypes sum of squares so although not adequate, is sufficient to allow meaningful conclusions to be drawn.

\section{TABLE 6}

Best fiting model: estimates of parameters Additive and dominance effects

\begin{tabular}{|c|c|c|c|c|c|}
\hline Chromosome & $\hat{d}$ & $\hat{h}$ & Chromosomes & $\hat{\imath}$ & $\hat{l}$ \\
\hline $1 \mathrm{~A}$ & $(+) 1 \cdot 34$ & 0.96 & $1 \mathrm{~A} \times 2 \mathrm{~B}$ & $-2 \cdot 87$ & \\
\hline $\mathrm{JB}$ & $(-) 0.24$ & 0.07 & $1 \mathrm{~A} \times 7 \mathrm{D}$ & $-0 \cdot 48$ & \\
\hline $1 \mathrm{D}$ & $(+) 0.12$ & $1 \cdot 02$ & $1 \mathrm{D} \times 6 \mathrm{~B}$ & 1.41 & -1.07 \\
\hline $2 \mathrm{~A}$ & $(+) 0.79$ & $1 \cdot 81$ & $2 \mathrm{~A} \times 3 \mathrm{~A}$ & $-0 \cdot 60$ & \\
\hline $2 \mathrm{~B}$ & $(+) 2 \cdot 18$ & $-1 \cdot 12$ & $2 \mathrm{~A} \times 4 \mathrm{~B}$ & $-7 \cdot 49$ & \\
\hline $2 D$ & (-) 0.67 & $1 \cdot 22$ & $2 \mathrm{~A} \times 5 \mathrm{~B}$ & $-2 \cdot 31$ & \\
\hline $3 \mathrm{~A}$ & $(+) 4.50 * *$ & -0.69 & $2 \mathrm{~A} \times 5 \mathrm{D}$ & $7 \cdot 12$ & \\
\hline $3 B$ & $(+) 3 \cdot 18 * *$ & $2 \cdot 00^{*}$ & $2 \mathrm{~A} \times 6 \mathrm{~B}$ & $-17 \cdot 66^{* * *}$ & \\
\hline $3 \mathrm{D}$ & $(-) 0.91$ & 0.92 & $2 \mathrm{~B} \times 6 \mathrm{~A}$ & $-2 \cdot 57$ & \\
\hline $4 \mathrm{~A}$ & $(+) 3.02 *$ & $2 \cdot 51 *$ & $2 \mathrm{~B} \times 6 \mathrm{~B}$ & $2 \cdot 80$ & \\
\hline $4 \mathrm{~B}$ & $(+) 4.57 *$ & $1 \cdot 34$ & $2 \mathrm{D} \times 3 \mathrm{~A}$ & 1.96 & \\
\hline $4 \mathrm{D}$ & $(-) 6 \cdot 82 * *$ & $-2 \cdot 14$ & $2 \mathrm{D} \times 4 \mathrm{~A}$ & $-7.57 *$ & \\
\hline $5 \mathrm{~A}$ & $(+) 3.07$ & $1 \cdot 73$ & $2 \mathrm{D} \times 5 \mathrm{~A}$ & $6.08 * *$ & \\
\hline $5 \mathrm{~B}$ & $(+) 1 \cdot 60$ & $-1 \cdot 42$ & $3 B \times 6 D$ & 0.82 & \\
\hline $5 \mathrm{D}$ & (-) 4.33 & $-1 \cdot 64$ & $3 \mathrm{D} \times 7 \mathrm{~B}$ & 0.62 & \\
\hline $6 \mathrm{~A}$ & $(-) 1 \cdot 34$ & -0.35 & $3 \mathrm{D} \times 7 \mathrm{D}$ & -1.06 & \\
\hline $6 \mathrm{~B}$ & $(-) 8 \cdot 41 * * *$ & $7 \cdot 80 * * *$ & $4 \mathrm{~A} \times 4 \mathrm{D}$ & $6 \cdot 19 * *$ & \\
\hline $6 \mathrm{D}$ & $(-) 0 \cdot 12$ & -1.03 & $4 \mathrm{~B} \times 4 \mathrm{D}$ & $-9 \cdot 80$ & \\
\hline $7 \mathrm{~A}$ & $(+) 0.02$ & $4 \cdot 74^{*}$ & $4 \mathrm{~B} \times 6 \mathrm{D}$ & -0.36 & \\
\hline $7 \mathrm{~B}$ & (-) 0.77 & -0.02 & $4 \mathrm{~B} \times 7 \mathrm{~A}$ & -5.95 & $-2 \cdot 23$ \\
\hline $7 \mathrm{D}$ & $(+) 1.90$ & $0 \cdot 08$ & $4 B \times 7 D$ & -0.97 & \\
\hline \multirow[t]{8}{*}{ Probabilitics } & \multicolumn{2}{|c|}{$*=0.05-0.01$} & $4 B \times 7 A$ & $1 \cdot 47$ & \\
\hline & \multicolumn{2}{|c|}{$* *=0.01-0.001$} & $5 \mathrm{~A} \times 7 \mathrm{~A}$ & $9 \cdot 23$ & \\
\hline & \multirow{6}{*}{\multicolumn{2}{|c|}{$* * *=<0.001$}} & $5 \mathrm{~A} \times 7 \mathrm{D}$ & $-4 \cdot 04$ & $-3 \cdot 87$ \\
\hline & & & $5 B \times 7 A$ & $-1 \cdot 01$ & \\
\hline & & & $5 \mathrm{D} \times 7 \mathrm{~A}$ & -6.09 & \\
\hline & & & $6 \mathrm{~A} \times 7 \mathrm{~A}$ & $3 \cdot 32$ & \\
\hline & & & $6 \mathrm{D} \times 7 \mathrm{~A}$ & $2 \cdot 36$ & \\
\hline & & & $6 \mathrm{D} \times 7 \mathrm{D}$ & -1.90 & \\
\hline
\end{tabular}

The estimates of the parameters obtained are shown in table 6 and reinforce the conclusions about the genetical architecture of height obtained from the earlier analyses. Here the sign in front of the $d$ cstimates indicates whether the increasing chromosome is carried by the taller parent Chinese Spring $(+)$ or by Cappelle-Desprez $(-)$. It is evident that there is almost complete dispersion of the increasing chromosomes between the parents and that dominance is in the direction of increasing height. Of the chromosomes exhibiting significant overdominance on the Triparental 2 tests only 7A now docs so, so that allowing for epistasis has removed the overdominance of the other chromosomes. Furthermore, 7A shows significant interactions 
with other chromosomes of the complement so it is likely that its overdominance would be removed by the inclusion of appropriate interaction parameters.

Finally, the significant estimates of the interactions are ambi-directional but overall there is a predominance of negative estimates which oppose the direction of dominance, indicating a duplicate type of gene interaction.

\section{Conventional analysis}

The family means of the parental, $\mathrm{F}_{1}, \mathrm{~F}_{2}$ and backcross generations are shown in table 7 and indicate that significant heterosis is exhibited in the $\mathrm{F}_{1}, \mathrm{~F}_{2}$ and backcross generations.

\section{TABLE 7}

Analysis of conventional generation means

\begin{tabular}{lc}
\multicolumn{1}{c}{ Generation } & $\begin{array}{c}\text { Mean height } \\
(\mathrm{cm})\end{array}$ \\
Chinese Spring & 107.27 \\
Cappelle-Desprez & 103.36 \\
$\mathbf{F}_{\mathbf{1}}$ & 119.36 \\
$\mathbf{F}_{\mathbf{2}}$ & 116.96 \\
$\mathbf{F}_{\mathbf{1}} \times$ Chinese Spring & 116.59 \\
$\mathbf{F}_{\mathbf{1}} \times$ Cappelle-Desprez & 113.28
\end{tabular}

$\begin{array}{ccc}\text { Model 1 } & \text { Model 2 } & \begin{array}{c}\text { Effects estimated } \\ \text { from best fitting } \\ \text { chromosome model }\end{array} \\ \hat{m}=107 \cdot 38 * * * & \hat{m}=114 \cdot 72 * * * & \hat{m}=108 \cdot 56 \\ (\hat{d})=2 \cdot 58 & (\hat{d})=2 \cdot 20 & (\hat{d})=2 \cdot 68 \\ (\hat{h})=13 \cdot 54 * * * & (\hat{h})=4 \cdot 62 * & (\hat{h})=17 \cdot 79 \\ & (\hat{\imath})=-9 \cdot 30 * * * & (\hat{\imath})=-20 \cdot 36 \\ \chi_{(3)}^{2}=26 \cdot 6 * * * & \chi_{(2)}^{2}=0 \cdot 85 & (\hat{l})=-7 \cdot 13 \\ & \text { Probabilities: } *=0 \cdot 05-0 \cdot 01, * * *=<0 \cdot 001 .\end{array}$

The genetical architecture of height in these crosses can be examined using the generation means analyses described by Mather and Jinks (1971). The between plot variances for the parental and $\mathrm{F}_{1}$ generations are homogeneous indicating that genotype-environment interactions are not an important component of the variation. However, the result of the $\mathrm{C}$ scaling test is $4.65 \pm 0.92$ showing that epistasis is present. This is further illustrated by the failure of an $m,(d)$ and $(h)$ model to account for the observed variation between family means on a joint scaling test: Model 1 , table 7. Models containing different combinations of epistatic parameters were fitted to these data and by allowing for $(i)$ type interactions alone a satisfactory fit is obtained: Model 2, table 7.

The estimates of these parameters allow the genetical architecture of height to be examined. The value of $(h)$ is positive indicating potence for increased height. Further, the heterosis exhibited is mirrored by $(h)>(d)$. Whether this is due to true overdominance cannot be elucidated from studying the means of these crosses although, as was shown earlier, overdominance for individual chromosomes does not occur when epistasis and 
gene dispersion are allowed for. Finally, the value of $(i)$ is negative and this again suggests a duplicate type of gene interaction.

It is interesting to compare the balanced sum of the individual additive, dominance and interaction effects, estimated from the whole chromosome analysis, with the balanced effects on the conventional model here.

These are obtained by summing the appropriate effects and, in the case of $(d)$ and $(i)$, allowing for the dispersion of the increasing chromosomes bctween the parents. These estimates are shown in table 7 . As can be seen, these values are similar to those obtained on the straight $m$, $(d)$ and $(h)$ model and indicate that the effects of epistasis have not been allowed for sufficiently in the chromosome model. These epistatic effects will be included in the estimate of $m$, and since they are expected to be predominantly negative, have deflated its estimate. This has also consequently deflated the estimate of $(i)$ and inflated the estimate of $(h)$ duc to the correlation of these effects with $m$, although $(d)$ is relatively unaffected since it is virtually independent of $m$ in its estimation.

\section{Discussion}

The details of the genetical architecture of height revealed by the whole chromosome analysis on the one hand and the conventional analysis on the other are very similar. In particular, both show that epistatic effects of an additive $\times$ additive type are an important component of the genetical control in this cross and that these act to oppose the additive effects. This type of genetical architecturc for height was also found by Law and Worland (1973a and $b$ ) and, in durum wheat, by Amaya, Busch and Lcbsock (1972), although othcr workers have found complementary interactions to be present (Chapman and McNeal, 1971), and also no epistasis (Bhatt, 1972) in other crosses. In all cases, however, dominance was towards loci increasing height.

The whole chromosome analysis rcveals a much greater complexity in the genetical control than the conventional analysis, however. It reveals that the hetcrosis exhibited can be explained by the dispersion of increasing chromosomes between the parents with directional dominance combined with the interactions which decrease the homozygous effects but do not affect the heterozygous effects of individual chrornosomes. This analysis also reveals that although both " nct" dominance and " net" interaction are strongly directional, there are, nevertheless, ambi-directional effects at the individual chromosome level.

The large epistatic component will have important consequences for selection for this charactcr since additive $\times$ additive interaction can be fixed in inbred lines which can be derived from this cross. These will act to reduce the amount of variation exhibited in the segregating generations and will thereby limit advances under selection.

Although the genetical architecture of height in the Hope into Chinese Spring substitution lines, described by Law and Worland (1973a and $b$ ), shows many similarities to the present analysis, the distribution of the genetical variation between chromosomes was somewhat different. First, most of the genetical variation with respect to height in the Hope set was carried by chromosomes of homoeologous groups 1, 5 and 6 . In the CappelleDesprez substitution lines large effects are again associated with groups 5 and 
6 but these chromosomes, apart from $6 \mathrm{~B}$, contribute mainly to the epistatic effects. Additive and dominance effects are mainly associated with chromosomes of homoeologous groups 3 and 4 . Secondly between chromosome interactions appeared to be restricted to within homoeologous groups only in the Hope set, whereas here between homoeologous group interactions also contribute to the variation to a significant extent.

The inability to find a whole chromosome model which fits the observed data makes it difficult to make definitive predictions about the consequences of new chromosomal combinations. However, tentative predictions can be made on the basis of the best fitting model.

The dispersion of the increasing chromosomes between the parents will result in transgressive segregation in the $\mathrm{F}_{2}$ and subsequent generations. Using computer simulation techniques (Fraser and Burnell, 1970) it can be shown that individuals taller than $135 \mathrm{~cm}(+25$ per cent heterosis) and shorter than $80 \mathrm{~cm}(-25$ per cent heterosis) would be expected at frequencies of 14 and 4 per 100 individuals in the $\mathrm{F}_{2}$ respectively in this experiment. In fact, the observed frequencies of individuals whose phenotypes were taller and shorter than these values was 37 and 6, respectively, out of the population of $607 \mathrm{~F}_{2}$ 's measured. These observed estimates are smaller than the expected but nevertheless demonstrate that \pm 25 per cent heterosis could have been predicted in the $\mathrm{F}_{2}$. However, it can also be predicted that the genotypes of the extreme inbred lines which could be derived from this cross will be $130 \mathrm{~cm}$ and $50 \mathrm{~cm}$. Thus the presence of duplicate interactions of an additive $\times$ additive type will reduce the limit to selection for increased height which may be below the expectations of the best $\mathrm{F}_{2}$ 's; although 50 per cent negative heterosis could be obtained.

Since large effects are associated with individual chromosomes it should be possible to increase the efficiency of selection for height in this cross by fixing certain chromosomes in the early generations. This could be achieved using crosses between the substitution lines, and between the substitution lines and Cappelle-Desprez. For example, if increased height was desired then the cross between the substitution line CS (Cappelle-Desprez 4D) and Cappelle-Desprez would fix this chromosome, which has a large positive effect on height, immediately. Similarly, if short straw was desired then the cross between substitution lines $2 \mathrm{~B}$ and $6 \mathrm{~B}$ would be expected to produce short segregants at a high frequency.

A final consideration of prediction concerns linkage. If linkage is an important component of the genetical architecture then the predictions as to the limits of selection must be regarded as minimal ones since they are based on estimates where the unit of inheritance is the chromosome. Thus if some chromosomes carry loci linked in the repulsion phase then greater advance may be realised by the exploitation of recombination between them.

Finally, the above results demonstrate the power of using chromosome substitution lines in elucidating the genetical architecture of quantitative characters of agronomic interest. The genetic nature of the heterosis for height in this cross has been established and has ruled out overdominance as a possible cause. This agrees with other in-depth analyses of the genetic nature of heterosis for height and other characters in Nicotiana (Jinks and Perkins, 1972; Jayasekara and Jinks, 1976). Although such studies have yet to be carried out for yield in wheat or other cereals, the authors see 
no reason to suppose that heterosis for this character will not have a similar genetic basis to that of height. The study of inter-varietal chromosome substitutions in wheat is well placed to establish this beyond doubt.

Acknowledgments. - We are grateful to Professor J. L. Jinks and Dr A. J. Wright for their comments on the manuscript.

\section{REFERENCES}

AKsr. , R. 1967. Quantitative genetic analysis of characters in wheat using chromosome substitution lines. (Theoretical considerations.) Genetics, 57, 192-211.

AMAYA, A. A., BUSCH, R. H., AND LEBSOCK, K. L. 1972. Estimates of genetic effects of heading date, plant height and grain yield in durum wheat. Crop Science, 12, 478-481.

внатт, G. м. 1972. Inheritance of heading date, plant height and kernel weight in two spring wheat crosses. Crop Science, 12, 95-97.

CHAPMAN, S. R., AND MGNEAL, F. I. 1971. Gene action for yield components and plant height in a spring wheat cross. Crop Science, 11, 384-386.

fraser, A. S., AND BURneld, D. 1970. Computer methods in Genetics. Mcgraw-Hill Lid.

JAYASEKARA, N. E. M., AND JINKS, J. L. 1976. Fffect of gene dispersion on estimates of components of generation ineans and variances. Heredity, 36, 31-40.

JINKs, J. L., AND PERKINS, J. M. 1972. Predicting the range of inbred lines. Heredity, 28 , 399-403.

LAW, c. N. 1966. Biometrical analysis using chromosome substitutions within a species. In Chromosome manipulation in Plant Genetics, ed. R. Riley and K. R. Lewis. Suppt. Heredity, 20, 59-85.

LAw, C. N. 1968. Genetic analysis using inter-varietal chromosome substitutions. Proc. 3rd Int. Wheat Genet. Symp., Canberra, 331-342.

LAW, c. N. 1972. The analysis of inter-varietal chromosome substitutions in wheat and their first generation hybrids. Heredity, 28, 169-179.

LAW, G. N., AND WORLAND, A. J. 1973a. Chromosome substitutions and their use in the analysis and prediction of wheat varietal performance. Proc. 4th Int. Wheat Genet. Symp., Missouri, 41-50.

LAW, C. N., AND WORLAND, A. J. 1973b. Aneuploidy in wheat and its uses in genetic analysis. Report of the Plant Breeding Institute for 1972, 25-65.

MATHER, K. 1967. Complementary and duplicate gene interactions in biometrical genetics. Heredity, 22, 97-102.

matiuer, K., AND Jinks, J. L. 1971. Biometrical Genetics. Chapman andi Hall Ltd., London. SNAPE, J. W., LAW, C. N., AND WORLAND, A. J. 1975. A method for the detection of epistasis in chromosome substitution lines of hexaploid wheat. Heredity, 34, 297-303.

VAN DER VEEN, J. H. 1959. Tests of non-allelic interaction and linkage for quantitative characters in generations derived from two diploid pure lines. Genetica, 30, 201-232. 